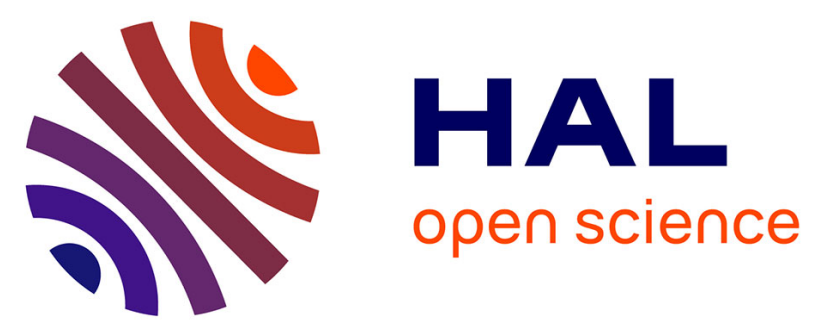

\title{
Observer-based Super Twisting Controller Robust to Wind Perturbation for Multirotor UAV
}

Hussein Hamadi, Benjamin Lussier, Isabelle Fantoni, Clovis Francis, Hassan Shraim

\section{- To cite this version:}

Hussein Hamadi, Benjamin Lussier, Isabelle Fantoni, Clovis Francis, Hassan Shraim. Observer-based Super Twisting Controller Robust to Wind Perturbation for Multirotor UAV. International Conference on Unmanned Aircraft Systems (ICUAS 2019), Jun 2019, Atlanta, GA, United States. pp.397-405, 10.1109/ICUAS.2019.8798307 . hal-02184578

\section{HAL Id: hal-02184578 https://hal.science/hal-02184578}

Submitted on 16 Jul 2019

HAL is a multi-disciplinary open access archive for the deposit and dissemination of scientific research documents, whether they are published or not. The documents may come from teaching and research institutions in France or abroad, or from public or private research centers.
L'archive ouverte pluridisciplinaire HAL, est destinée au dépôt et à la diffusion de documents scientifiques de niveau recherche, publiés ou non, émanant des établissements d'enseignement et de recherche français ou étrangers, des laboratoires publics ou privés. 


\title{
Observer-based Super Twisting Controller Robust to Wind Perturbation for Multirotor UAV
}

\author{
Hussein Hamadi ${ }^{1,2}$, Benjamin Lussier ${ }^{1}$, Isabelle Fantoni ${ }^{1,3}$, Clovis Francis ${ }^{2}$ and Hassan Shraim ${ }^{2}$
}

\begin{abstract}
Control design for multi rotors UAV is an important challenge for engineers and scientists, due to the fact that the standard configurations are under-actuated, highly nonlinear, and unstable systems. In this paper, a wind force compensation strategy is proposed for a quadrotor. This strategy relies on a second order sliding mode controller based on the super twisting algorithm (STA) with an observer. Second order sliding mode technique ensures robustness to external disturbances and time varying, parametric and nonlinear uncertainties. Integration of an observer in the closed-loop system is needed for states reconstruction and the estimation of unknown external forces such as the wind effect. This estimation will allow a better monitoring of the system's status than passive robustness, providing the opportunity for recovery tactics such as an emergency landing when the external perturbations become too strong for the system. The effectiveness of the proposed strategy is compared to an adaptative gain controller through simulation and validated in real experiments on a quadrotor.
\end{abstract}

\section{INTRODUCTION}

Over the last decade, there has been a growing interest in flying machines without on board human pilots known as UAVs (Unmanned Aerial Vehicles). The application scope of UAVs ranges from homeland security (coast guard, borders protections...) and disaster relief (exploring unknown environment, monitoring or intervening in potentially dangerous areas...) to weather forecasting [1], [2]. Since in outdoors environment the drone is exposed to adverse atmospheric conditions, reliable and robust control algorithms are required.

In the literature, a large number of publications on multicopters addresses this control problem. Some of these works propose the development of linear controllers like PID and LQ regulators [3] [4], while others suggest the development of nonlinear methods to maintain the stability of the system. Among the latter, we can list the backstepping control approach [5], fuzzy controllers [6] and sliding mode approaches [7].

However, there exist relatively few works where wind effects on these systems are quantified. In [8], the authors developed two methods to estimate the wind speed and its direction. In a direct approach, wind information are

\footnotetext{
${ }^{1}$ Sorbonne Universités, Université de Technologie de Compiègne, CNRS, UMR 7253 Heudiasyc, 60200 Compiègne, France, Email: \{hussein.hamadi, benjamin.lussier\}ahds.utc.fr

${ }^{2}$ Université Libanaise, Faculté de Génie, Centre de Recherche Scientifique en Ingénierie (CRSI), Liban, Email: \{cfrancis, hassan.shraim\}@ul.edu. lb

${ }^{3}$ Laboratoire des Sciences du Numérique de Nantes (LS2N), UMR CNRS 6004, 1 rue de la Noë, 44321 Nantes, France, Email: \{isabelle.fantoni\}els 2 n.fr
}

estimated using a wind sensor (anemometer) mounted on top of the vehicle. In an indirect approach, the attitude data from the drone are used in the estimation. However, the wind effect is considered as only due to the air frame drag which does not reflect real flight conditions. The problem of wind estimation was also addressed in [9], where an extended state observer is developed to identify wind disturbances, but only indoor experiments where conducted to validate this method. A linear observer for the estimation of wind disturbances is presented in [10]. Real-time outdoor experiments where conducted using a GPS receiver to test the proposed method in real flight conditions. Nevertheless, the authors explain that it can only be used near the equilibrium point, i.e. hovering, since it is based on a linear model of the vehicle.

Indeed, the design of a robust control law against disturbances due to wind is a difficult problem [11], especially when tracking aggressive trajectories. Thus two approaches are oftenly used: first the implicit modeling of the forces of wind on the drone and its integration in the expression of the control law, and second the use of a robust controller to tolerate wind effects and other uncertainties (such as model uncertainties).

Among the first approach, Sydney et al. [12] carried out an estimation process of the wind effect on a quadrotor and used it to design a control law capable of countering its disturbances. The authors in [13] investigated the wind effect on a quadrotor model, and showed that it can be viewed as an oblique flow approaching the rotors based on the bladeelement momentum theory. Huan et al. [14] took into account the aerodynamic effect by proposing a law of aggressive command for a quadrotor using the blade flapping approach. In [15], an active rejection of wind disturbance using an approximate feedback linearization of the model dynamics is proposed and validated. However, such a controller based on feedback linearization is known to be very sensitive to sensor noise, since up to three-order derivatives of the states are included in the inputs.

Other works, such as [16] and [17] have experimentally validated their contribution. In particular in [17], three types of disturbances including payloads, rotors failures, and the wind generated by a fan are chosen separately to verify the effectiveness of the controller in each situation.

Among works of the second approach, [18] and [19], propose robust control laws with adaptive gains to ensure the stability of the system. In [20], the authors address flight control in presence of wind-gust disturbances during trajectory tracking with a 6DOF nonlinear dynamics model separated into two subsystems (dynamic and kinematic). 
A hierarchical controller is used to stabilize the underactuated subsystems using the sliding mode and the adaptive control techniques to deal with slow and fast varying wind conditions. At the same time, a backstepping technique is used to stabilize the inner loop heading dynamics.

The authors of [21] developed an adaptive robust controller based on the sliding mode algorithm to deal with payload variation and wind gust disturbances. To quantify the wind impact, they implemented a propeller momentum drag and wind gust model including forces and moment disturbances. In our work, we propose a full scheme of wind estimation and rejection. Such a closed loop control method based on active disturbance detection and validated on real experiments in outdoor environment is in our opinion an original contribution compared to the presented litterature. Through active detection, our proposed strategy helps first to detect wind condition that would be too difficult to the UAV (allowing to deploy recovery mechanisms such as a parachute) and second to better coordinate with fault tolerance mechanisms such as detection and recovery of failing motors. Through a passive approach like those presented in the literature,, the robustness of adaptative controllers usually allows the second but not the first.

In this paper, a second order sliding mode technique based on the Super-Twisting Algorithm (STA) is used as a control law which is able to deal with matched uncertainties such as imperfections in the model's parameter estimation (mass, inertias, motors constants...) and bounded external disturbances [22]. The induced aerodynamic forces representing the unknown external wind disturbances are estimated via a first order differentiator also based on the STA [23]. The main contribution of this paper is to propose a guided Sliding Mode Controller (SMC) that uses a wind observer to take into account wind disturbances, following the approach of explicitly taking into account wind perturbations. Compared to passive robust techniques, we consider that this approach could give better results in extreme conditions, but would also allow the system to identify dangerous situations regarding wind perturbations and to perform recovery actions like dropping its altitude or even landing to avoid possible control loss.

This paper is organized as follows: section II presents the nonlinear dynamic model of the quadrotor. Based on this model, a STA control law is developed in section III. In section IV, we present a robust observer designed to estimate the external disturbances (and particularly the wind forces). Section V compares our approach to a robust adaptative controller in simulations and details a validation of the proposed controller and observer using real experiments. Finally, some conclusions and perspectives are presented in section VI.

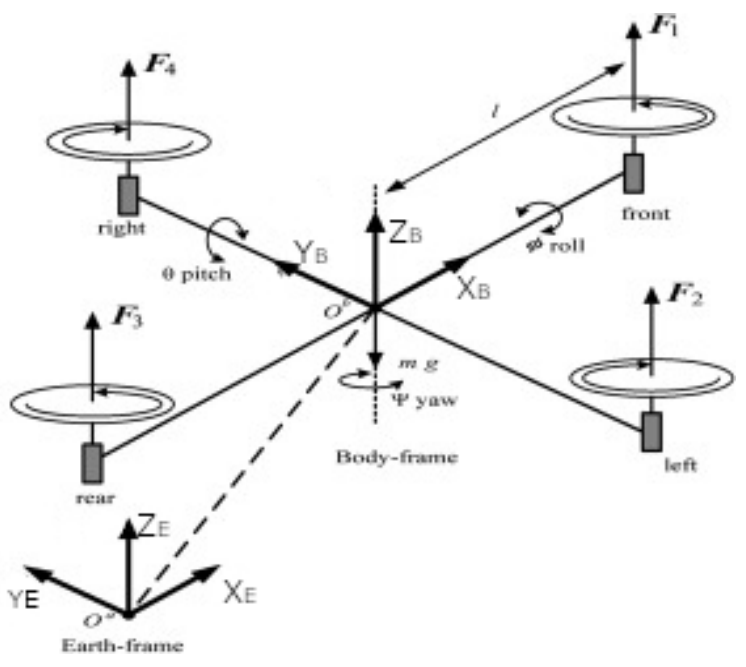

Fig. 1: The quadrotor and the reference frames

\section{EQUATIONS OF MOTION}

The dynamics of a quadrotor aircraft, depicted in Figure 1 , is modeled by the following equations:

$$
\begin{aligned}
& m \ddot{\xi}=-m g_{g} Z_{E}+R u_{t} Z_{E}+F_{p} \\
& I \dot{\eta}=-\operatorname{Skew}(\eta) I \eta+G+\tau+M_{r}
\end{aligned}
$$

where the Skew function is the Skew-symmetric matrix of the vector, $\xi=\left[\begin{array}{lll}x & y & z\end{array}\right]^{T}$ and $\eta=\left[\begin{array}{lll}p & q & r\end{array}\right]^{T}$ are respectively the vehicle position in Earth-frame and angular velocities in Body-frame, $m$ is the mass of the quadrotor, $I=\operatorname{diag}\left(I_{x}, I_{y}, I_{z}\right)$ is the inertia matrix of the rigid body, $g_{g}$ is the gravitational constant, $u_{t}$ and $\tau=\left[\begin{array}{lll}\tau_{\phi} & \tau_{\theta} & \tau_{\psi}\end{array}\right]^{T}$ represent the virtual control inputs of the system (thrust and torques respectively) detailed in (9), $F_{p}=\left[\begin{array}{lll}F_{x} & F_{y} & F_{z}\end{array}\right]^{T}$ and $M_{r}=\left[\begin{array}{lll}M_{\phi} & M_{\theta} & M_{\psi}\end{array}\right]^{T}$ are the induced aerodynamic forces and moments acting on the quadrotor caused by external wind and gusts. It is worth mentioning that the aerodynamic moments $M_{r}$ and the aerodynamic force $F_{z}$ are named matched disturbances since they affect the system states which are directly controlled by the motors, while only the forces $F_{x}$ and $F_{y}$ are unmatched ones [24]. For this reason, we will only consider $F_{x}$ and $F_{y}$ in our wind observer, as a sliding mode controller is already robust against matched uncertainties. Finally, $G=\left[\begin{array}{lll}G_{\phi} & G_{\theta} & G_{\psi}\end{array}\right]^{T}$ represents the gyroscopic effect due respectively to body rotation and propellers orientation changes, and $R$ is the rotation matrix from the Body-frame to the Earth-frame given by:

$$
R=\left(\begin{array}{ccc}
C_{\psi} C_{\theta} & C_{\psi} S_{\phi} S_{\theta}-C_{\phi} S_{\psi} & C_{\phi} C_{\psi} S_{\theta}+S_{\phi} S_{\psi} \\
S_{\psi} C_{\theta} & S_{\phi} S_{\psi} S_{\theta}+C_{\phi} C_{\psi} & C_{\phi} C_{\psi} S_{\theta}-S_{\phi} S_{\psi} \\
-S_{\theta} & C_{\theta} S_{\phi} & C_{\phi} C_{\theta}
\end{array}\right)
$$

where $S$. and $C$. denote respectively the $\sin ()$ and $\cos ()$ functions. Thus, we obtain the full mathematical model:

\section{1) Position dynamic model:}

$$
\left(\begin{array}{c}
m \ddot{x} \\
m \ddot{y} \\
m \ddot{z}
\end{array}\right)=\left(\begin{array}{c}
0 \\
0 \\
-m g_{g}
\end{array}\right)+\left(\begin{array}{c}
C_{\phi} C_{\psi} S_{\theta}+S_{\phi} S_{\psi} \\
C_{\phi} S_{\psi} S_{\theta}-S_{\phi} C_{\psi} \\
C_{\theta} C_{\phi}
\end{array}\right) u_{t}+\left(\begin{array}{c}
F_{x} \\
F_{y} \\
F_{z}
\end{array}\right)
$$


2) Attitude dynamic model:

$\left(\begin{array}{c}I_{x} \dot{p} \\ I_{y} \dot{q} \\ I_{z} \dot{r}\end{array}\right)=\left(\begin{array}{c}\left(I_{y}-I_{z}\right) q r \\ \left(I_{z}-I_{x}\right) p r \\ \left(I_{x}-I_{y}\right) p q\end{array}\right)+\left(\begin{array}{c}-J_{r} q \Omega \\ J_{r} p \Omega \\ 0\end{array}\right)+\left(\begin{array}{c}\tau_{\phi} \\ \tau_{\theta} \\ \tau_{\psi}\end{array}\right)+\left(\begin{array}{c}M_{\phi} \\ M_{\theta} \\ M_{\psi}\end{array}\right)$

where $J_{r}$ is the rotor inertia and $\Omega$ is the overall residual motors speed from the unbalanced rotor rotation (5).

$$
\Omega=\varpi_{1}+\varpi_{3}-\varpi_{2}-\varpi_{4}
$$

where $\varpi_{i}$ are propeller angular speeds. The transformation matrix $T_{\eta}$ relating to body angular velocities $\left[\begin{array}{lll}p & q & r\end{array}\right]^{T}$ and Euler angular rates $\left[\begin{array}{lll}\dot{\phi} & \dot{\theta} & \dot{\psi}\end{array}\right]^{T}$ is given by:

$$
\begin{array}{r}
\left(\begin{array}{c}
p \\
q \\
r
\end{array}\right)=T_{\eta}\left(\begin{array}{c}
\dot{\phi} \\
\dot{\theta} \\
\dot{\psi}
\end{array}\right) \\
T_{\eta}=\left(\begin{array}{ccc}
1 & 0 & -S_{\theta} \\
0 & C_{\phi} & C_{\theta} S_{\phi} \\
0 & -S_{\phi} & C_{\theta} C_{\phi}
\end{array}\right)
\end{array}
$$

Considering that the UAV will not do aggressive maneuvers but only small roll and pitch angles variations (Assumption 5 below), we can assume that $T_{\eta}$ is very close to the identity matrix $T_{\eta}=I_{3 x 3}$. Thus the attitude dynamics model (4) can be simplified as:

$$
\left(\begin{array}{c}
I_{x} \ddot{\phi} \\
I_{y} \ddot{\theta} \\
I_{z} \ddot{\psi}
\end{array}\right)=\left(\begin{array}{l}
\left(I_{y}-I_{z}\right) \dot{\theta} \dot{\psi} \\
\left(I_{z}-I_{x}\right) \dot{\phi} \dot{\psi} \\
\left(I_{x}-I_{y}\right) \dot{\phi} \dot{\theta}
\end{array}\right)+\left(\begin{array}{c}
-J_{r} \dot{\theta} \Omega \\
J_{r} \dot{\phi} \Omega \\
0
\end{array}\right)+\left(\begin{array}{c}
\tau_{\phi} \\
\tau_{\theta} \\
\tau_{\psi}
\end{array}\right)+\left(\begin{array}{c}
M_{\phi} \\
M_{\theta} \\
M_{\psi}
\end{array}\right)
$$

The flight of the quadrotor is driven by multiple propellers. The propeller angular speeds $\varpi_{i}, i=1,2,3,4$ will determine the total thrust $u_{t}$ and moments $\tau$. According to the Figure 1's rotor numbering, the total thrust and torques representing the input vector of the system is given by:

$$
\begin{aligned}
& u_{t}=F_{1}+F_{2}+F_{3}+F_{4} \\
& \tau_{\phi}=\left(F_{4}-F_{2}\right) * l \\
& \tau_{\theta}=\left(F_{3}-F_{1}\right) * l \\
& \tau_{\psi}=\left(\tau_{1}+\tau_{3}\right)-\left(\tau_{2}+\tau_{4}\right)
\end{aligned}
$$

More information about the derivation of the dynamic model and the expressions of the control inputs are given in [25].

\section{CONTROL LAW}

The control law design is performed under several assumptions:

Assumption 1 The reference trajectory controllers are continuous, differentiable and their derivatives are bounded.

Assumption 2 The external disturbances and their first derivatives are bounded. In other words, the wind forces variations are considered continuous.

Assumption 3 The roll, pitch and yaw angles are constrained to $(-\pi / 2<\phi, \theta<\pi / 2)$ and $(-\pi<\psi<\pi)$, so acrobatic behaviors such as looping are not allowed.
Assumption 4 The structure of the system is supposed to be rigid and symmetrical.

Assumption 5 The system dynamics are limited to small angles and small variations of linear and angular velocities. This behavior is current in practice with UAV.

Assumption 6 All the control inputs are bounded, i.e., $\left.\left.u_{t} \in\right] 0,1\right]$ and $\tau_{\phi}, \tau_{\theta}, \tau_{\psi} \in[-1 ; 1]$

The objective of the controller is to ensure the convergence of the actual state vector $[x, y, z, \psi]$ to the desired trajectories $\left[x_{r}, y_{r}, z_{r}, \psi_{r}\right]$. Let us first consider the following vectors: $x_{1}=[z, \phi, \theta, \psi]^{T}$, and $x_{2}=[\dot{z}, \dot{\phi}, \dot{\theta}, \dot{\psi}]^{T}$ so we end up with the following state-space form of the model:

$$
\left\{\begin{array}{l}
\dot{x}_{1}=x_{2} \\
\dot{x}_{2}=f\left(x_{1}, x_{2}\right)+g\left(x_{1}, x_{2}\right) u+w(t)
\end{array}\right.
$$

where $w(t)=\left[\begin{array}{llll}w_{z} & w_{\phi} & w_{\theta} & w_{\psi}\end{array}\right]^{T}$ represents the external disturbances vector. The vector $f\left(x_{1}, x_{2}\right)$, the matrix $g\left(x_{1}, x_{2}\right)$ and $u$ are defined as:

$$
\begin{aligned}
& f\left(x_{1}, x_{2}\right)=\left(\begin{array}{c}
-g_{g} \\
\left(I_{y}-I_{z}\right) \dot{\theta} \dot{\psi}-J_{r} \dot{\theta} \Omega \\
\left(I_{z}-I_{x}\right) \dot{\phi} \dot{\psi}+J_{r} \dot{\phi} \Omega \\
\left(I_{x}-I_{y}\right) \dot{\phi} \dot{\theta}
\end{array}\right) \\
& u=\left(\begin{array}{c}
u_{t} \\
\tau_{\phi} \\
\tau_{\theta} \\
\tau_{\psi}
\end{array}\right) \\
& g\left(x_{1}, x_{2}\right)=\left[\begin{array}{cccc}
\frac{1}{m}\left(C_{\theta} C_{\phi}\right) & 0 & 0 & 0 \\
0 & \frac{1}{I_{x}} & 0 & 0 \\
0 & 0 & \frac{1}{I_{y}} & 0 \\
0 & 0 & 0 & \frac{1}{I_{z}}
\end{array}\right]
\end{aligned}
$$

Note that in (11) the terms $J_{r} \dot{\phi} \Omega$ and $-J_{r} \dot{\theta} \Omega$ will be neglected by means of the (Assumption 5) in the sequel. Thus according to the assumptions cited before, the control matrix $g\left(x_{1}, x_{2}\right)$ is non-singular and thus invertible, and the following inequalities are established:

$$
\begin{aligned}
& \left|f_{i}\left(x_{1}, x_{2}\right)\right|<\tilde{f}_{i}, i=1, \ldots, 4 \\
& \left|\dot{w}_{i}\right|<\tilde{w}_{i}, \quad i=z, \phi, \theta, \psi
\end{aligned}
$$

where $\tilde{f}_{i}$ and $\tilde{w}_{i}$ are known positive values. The sliding manifolds vector $s=\left[\begin{array}{llll}s_{z} & s_{\phi} & s_{\theta} & s_{\psi}\end{array}\right]^{T}$ is defined as:

$$
s=\dot{e}+\lambda e
$$

where $e=\left[\begin{array}{llll}e_{z} & e_{\phi} & e_{\theta} & e_{\psi}\end{array}\right]^{T}$ represent the errors between the actual states of the system and the desired ones and $\lambda=\operatorname{diag}\left(\lambda_{z}, \lambda_{\phi}, \lambda_{\theta}, \lambda_{\psi}\right)$ is a positive definite gain matrix. According to [22], we choose the control input as the following expression:

$$
\begin{aligned}
u= & g\left(x_{1}, x_{2}\right)^{-1}\left(\ddot{x}_{1 d}-\lambda \dot{e}-K_{1} \sqrt{|s|} \operatorname{sgn}(s)\right. \\
& \left.-K_{2} \int_{0}^{t} \operatorname{sign}(s(\epsilon)) d \epsilon-f\left(x_{1}, x_{2}\right)\right)
\end{aligned}
$$

where $\ddot{x}_{1 d}$ is the desired acceleration provided by the trajectory generator, $K_{1}=\operatorname{diag}\left(K_{1 z}, K_{1 \phi}, K_{1 \theta}, K_{1 \psi}\right)$ and 


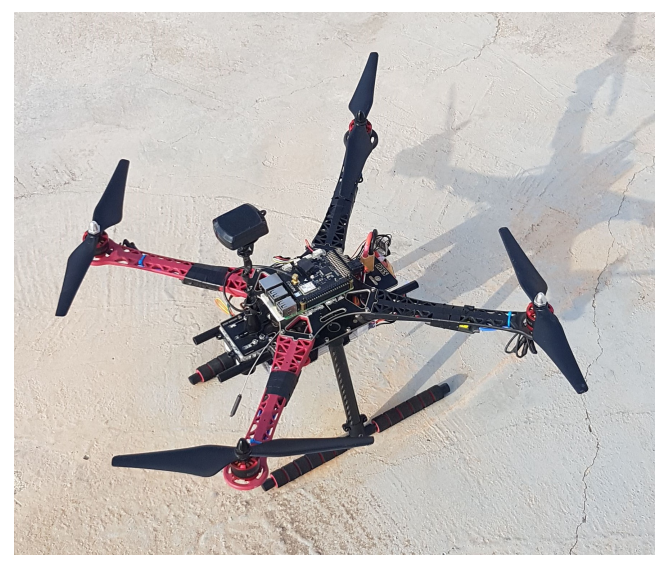

Fig. 2: Experimental quadrotor

$K_{2}=\operatorname{diag}\left(K_{2 z}, K_{2 \phi}, K_{2 \theta}, K_{2 \psi}\right)$ are super twisting control positive gain matrices, and $\operatorname{sign}($.$) is the sign function. Thus$ we obtain the following closed-loop error dynamics:

$$
\dot{s}=-K_{1} \sqrt{|s|} \operatorname{sign}(s)-K_{2} \int_{0}^{t} \operatorname{sign}(s(\epsilon)) d \epsilon+w(t)
$$

To prove the stability and the time convergence of this system, we define the variable $\zeta=\left[\zeta_{1}, \zeta_{2}\right]^{T}=$ $\left[s,-K_{2} \int_{0}^{t} \operatorname{sign}(s) d \epsilon\right]^{T}$, then the equation (17) can be rewritten as:

$$
\begin{aligned}
& \dot{\zeta}_{1}=-K_{1} \sqrt{\left|\zeta_{1}\right|} \operatorname{sign}\left(\zeta_{1}\right)+\zeta_{2} \\
& \dot{\zeta}_{2}=-K_{2} \operatorname{sign}\left(\zeta_{1}\right)+\dot{w}
\end{aligned}
$$

The proof of finite convergence of the variables $\zeta_{1}$ and $\zeta_{2}$ follows the same procedure as in [22]. It is worth nothing that the translational motion in the $x$-axis and $y$-axis depends on the pitching and rolling torques, so the development of the control inputs is divided into an altitude and heading control upon which depends an X-Y translational motion control.

\section{A. Altitude and heading control}

We define the altitude tracking error, which is the difference between the desired and the measured altitudes as follows:

$$
e_{z}=z_{r}-z
$$

We also define the sliding variables for these errors:

$$
s_{z}=\dot{e}_{z}+\lambda_{z} e_{z}
$$

In this case, the dynamics of the altitude sliding variable $s_{z}$ is given by:

$$
\dot{s}_{z}=\ddot{e}_{z}+\lambda_{z} \dot{e}_{z}
$$

By substituting (3) and (19) into (21), we obtain:

$$
\dot{s}_{z}=\ddot{z}_{r}+g_{g}-\frac{\left(C_{\theta} C_{\phi}\right)}{m} u_{t}-w_{z}+\lambda_{z} \dot{e}_{z}
$$

As a manner of fact, the control input $u_{t}$ is composed of two parts: a continuous part called equivalent input, and a discontinuous part based on the super twisting algorithm (23).

$$
u_{t}=u_{t e q}+u_{t d i s}
$$

The equivalent input $u_{t e q}$ is computed by applying the sliding condition, $\dot{s}=0$, and the discontinuous part $u_{t d i s}$ is derived in order to obtain the closed-loop error dynamics as given in (17) in each subsystem. By applying the sliding condition to the altitude i.e. $\dot{s}_{z}=0$, we obtain from (21) a sliding property that we want $u_{t e q}$ to satisfy:

$$
\ddot{z}_{r}+g_{g}-\frac{\left(C_{\theta} C_{\phi}\right)}{m} u_{t e q}-w_{z}+\lambda_{z} \dot{e}_{z}=0
$$

As the matched uncertainties $w_{z}$ will be tolerated by the super twisting controller, we thus choose for the equivalent input:

$$
u_{t e q}=\frac{m}{\left(C_{\theta} C_{\phi}\right)}\left(\ddot{z}_{r}+g_{g}+\lambda_{z} \dot{e}_{z}\right)
$$

and as given by the super twisting algorithm, we choose for the discontinuous part:

$$
u_{t d i s}=-K_{1 z} \sqrt{\left|s_{z}\right|} \operatorname{sign}\left(s_{z}\right)-K_{2 z} \int_{0}^{t} \operatorname{sign}\left(s_{z}(\epsilon)\right) d \epsilon
$$

By adding the equivalent term and the discontinuous term, the full expression of the control input becomes:

$$
\begin{aligned}
u_{t} & =\frac{m}{\left(C_{\theta} C_{\phi}\right)}\left(\ddot{z}_{r}+g_{g}+\lambda_{z} \dot{e}_{z}\right) \\
& -K_{1 z} \sqrt{\left|s_{z}\right|} \operatorname{sign}\left(s_{z}\right)-K_{2 z} \int_{0}^{t} \operatorname{sign}\left(s_{z}(\epsilon)\right) d \epsilon
\end{aligned}
$$

By choosing the altitude control gains as follows:

$$
K_{1 z}=\frac{m K_{1 z}^{\prime}}{\left(C_{\theta} C_{\phi}\right)} \quad K_{2 z}=\frac{m K_{2 z}^{\prime}}{\left(C_{\theta} C_{\phi}\right)}
$$

and by substituting (28) into (27), then finally by substituting the control law (27) in (22), the dynamics of $s_{z}$ becomes:

$$
\dot{s}_{z}=-K_{1 z}^{\prime} \sqrt{\left|s_{z}\right|} \operatorname{sign}\left(s_{z}\right)-K_{2 z}^{\prime} \int_{0}^{t} \operatorname{sign}\left(s_{z}(\epsilon)\right) d \epsilon+w_{z}
$$

Which satisfy having the same form as the equation (17). The same procedure is used to obtain the yaw torque:

$$
\begin{aligned}
\tau_{\psi} & =I_{z}\left(\ddot{\psi}_{r}-\frac{M_{\psi}}{I_{z}}+\lambda_{\psi} \dot{e}_{\psi}-\frac{\left(I_{x}-I_{y}\right)}{I_{z}} \dot{\phi} \dot{\theta}\right) \\
& -K_{1 \psi} \sqrt{\left|s_{\psi}\right|} \operatorname{sign}\left(s_{\psi}\right)-K_{2 \psi} \int_{0}^{t} \operatorname{sign}\left(s_{\psi}(\epsilon)\right) d \epsilon
\end{aligned}
$$

\section{B. Translational motion in $X-Y$ direction}

In this subsection, the procedure to derive the roll and pitch torques is introduced. The first challenge is to obtain the desired roll and pitch variables from the position dynamic model (3). We define the following tracking errors:

$$
\begin{aligned}
& e_{x}=x_{r}-x \\
& e_{y}=y_{r}-y
\end{aligned}
$$

By applying the sliding conditions, i.e. $\dot{s}_{x}=0, \dot{s}_{y}=0$, we get:

$$
\begin{aligned}
& \ddot{x}_{r}-\frac{\left(u_{t}\right)}{m} u_{x e q}-\frac{F_{x}}{m}+\lambda_{x} \dot{e}_{x}=0 \\
& \ddot{y}_{r}-\frac{\left(u_{t}\right)}{m} u_{y e q}-\frac{F_{y}}{m}+\lambda_{y} \dot{e}_{y}=0
\end{aligned}
$$


Where the wind forces $F_{x}$ and $F_{y}$ represent the only unmatched uncertainties in the terms $w_{x}$ and $w_{y}$. The terms $u_{x e q}$ and $u_{y e q}$ are given by:

$$
\begin{aligned}
& u_{x e q}=C_{\phi} C_{\psi} S_{\theta}+S_{\phi} S_{\psi} \\
& u_{y e q}=C_{\phi} S_{\psi} S_{\theta}-S_{\phi} C_{\psi}
\end{aligned}
$$

Following the same procedure as in the previous section, we define:

$$
\begin{aligned}
\frac{u_{t}}{m} u_{x} & =\ddot{x}_{r}-\frac{F_{x}}{m}+\lambda_{x} \dot{e}_{x} \\
& -K_{1 x} \sqrt{\left|s_{x}\right|} \operatorname{sign}\left(s_{x}\right)-K_{2 x} \int_{0}^{t} \operatorname{sign}\left(s_{x}(\epsilon)\right) d \epsilon \\
\frac{u_{t}}{m} u_{y} & =\ddot{x}_{r}-\frac{F_{y}}{m}+\lambda_{y} \dot{e}_{y} \\
& -K_{1 y} \sqrt{\left|s_{y}\right|} \operatorname{sign}\left(s_{y}\right)-K_{2 y} \int_{0}^{t} \operatorname{sign}\left(s_{y}(\epsilon)\right) d \epsilon
\end{aligned}
$$

The desired roll and pitch can be determined from $u_{x}$ and $u_{y}$ and by using the desired heading:

$$
\begin{aligned}
& \phi_{r}=\arcsin \left(S_{\psi_{r}} u_{x}-C_{\psi_{r}} u_{y}\right) \\
& \theta_{r}=\arcsin \left(\frac{C_{\psi_{r}} u_{x}+S_{\psi_{r}} u_{y}}{C_{\phi_{r}}}\right)
\end{aligned}
$$

Thus, we can define the roll and pitch errors by:

$$
\begin{aligned}
& e_{\phi}=\phi_{r}-\phi \\
& e_{\theta}=\theta_{r}-\theta
\end{aligned}
$$

These values are used in order to calculate the appropriate torques as follows:

$$
\begin{aligned}
\tau_{\phi}= & I_{x}\left(\ddot{\phi}_{r}-\frac{M_{\phi}}{I_{x}}+\lambda_{\phi} \dot{e}_{\phi}-\frac{\left(I_{y}-I_{z}\right)}{I_{x}} \dot{\theta} \dot{\psi}\right. \\
& -K_{1 \phi} \sqrt{\left|s_{\phi}\right|} \operatorname{sign}\left(s_{\phi}\right)-K_{2 \phi} \int_{0}^{t} \operatorname{sign}\left(s_{\phi}(\epsilon)\right) d \epsilon \\
\tau_{\theta}= & I_{y}\left(\ddot{\theta}_{r}-\frac{M_{\theta}}{I_{y}}+\lambda_{\theta} \dot{e}_{\theta}-\frac{\left(I_{z}-I_{x}\right)}{I_{y}} \dot{\phi} \dot{\psi}\right. \\
& -K_{1 \theta} \sqrt{\left|s_{\theta}\right|} \operatorname{sign}\left(s_{\theta}\right)-K_{2 \theta} \int_{0}^{t} \operatorname{sign}\left(s_{\theta}(\epsilon)\right) d \epsilon
\end{aligned}
$$

Based on the Assumption 5, we can ignore the desired linear and angular accelerations as well as the desired angular velocities in the control inputs expressions.

\section{OBSERVER DESIGN}

Since the wind induced forces $F_{x}$ and $F_{y}$ are used to derive the control law in (34), we estimate them via a super-twisting observer. We only develop the observer for the estimation of $F_{x}$ and $F_{y}$ components since they are not compensated directly by the control law unlike $F_{z}$ and the moment disturbances, as previously explained in Section II.

\section{A. Nonlinear observer and differentiator}

In order to use the approach in [26] and [27] for the estimation of the unknown wind forces $F_{x}$ and $F_{y}$ using the available sensor measurements, we put the $\mathrm{x}$-direction state model under the form:

$$
\left\{\begin{array}{l}
\dot{x}=V_{x} \\
m \dot{V}_{x}=\hat{u}_{x} u_{t}+F_{x}
\end{array}\right.
$$

The goal is to develop a virtual control law $u_{x}$ which is able to stabilize the sliding manifold $\sigma$ by driving $\sigma, \dot{\sigma} \longrightarrow 0$, and to cancel out any drift terms or disturbances. To do this, we choose $\sigma$ as follows

$$
\sigma_{x}=V_{x}-V_{x d}=0
$$

or

$$
\sigma_{x}=V_{x}-K_{p}\left(x-x_{d}\right)=0
$$

where $V_{x}$ is the velocity in the x-direction, $V_{x d}$ is the desired velocity and $K_{p}=1$ is the tracking gain. This command will be followed by the virtual control law to allow the vehicle to track the desired trajectory. Moreover the $(\sigma)$ dynamics is of relative degree 1 , since

$$
\dot{\sigma}_{x}=\frac{u_{t}}{m} \hat{u}_{x}+\frac{F_{x}(t)}{m}-K_{p}\left(V_{x}-V_{x d}\right)
$$

is under the form

$$
\dot{\sigma}=\frac{u_{t}}{m} \hat{u}_{x}+b_{x}(t)
$$

where $b_{x}(t)$ represents the uncertain function in $\mathrm{x}$-direction. Its first derivative is given by:

$$
\dot{b}_{x}(t)=\frac{\dot{F}_{x}(t)}{m}-K_{p}\left(\dot{V}_{x}-\dot{V}_{x d}\right)
$$

We assume that the wind forces (and particularly $F_{x}(t)$ and $\left.F_{y}(t)\right)$ are continuous and differentiable anytime, thus its derivative has a Lipschitz constant by assuming that the disturbances and their derivative are bounded i.e. $\left|F_{x}(t)\right|<$ $F_{x}^{L I M}$ and $\left|\dot{F}_{x}\right|<\dot{F}_{x}^{L I M}$. Then the function $b_{x}(t)$ is also differentiable and has a Lipschitz constant $L_{x}$. The term $F_{x}$ is estimated by defining the following system:

$$
\left\{\begin{array}{l}
\dot{z}_{0 x}=v_{0 x}+\frac{u_{t}}{m} u_{x}-K_{p}\left(\dot{x}-\dot{x}_{d}\right) \\
v_{0 x}=-\alpha_{0 x}\left|z_{0 x}-\sigma_{x}\right|^{2 / 3} \operatorname{sign}\left(z_{0 x}-\sigma_{x}\right)+z_{1 x} \\
\dot{z}_{1 x}=v_{1 x} \\
v_{1 x}=-\alpha_{1 x}\left|z_{1 x}-v_{0 x}\right|^{1 / 2} \operatorname{sign}\left(z_{1 x}-v_{0 x}\right)+z_{2 x} \\
\dot{z}_{2 x}=-\alpha_{2 x} \operatorname{sign}\left(z_{2 x}-v_{1 x}\right) \\
\hat{F}_{x} / m=z_{1 x}
\end{array}\right.
$$

where $\dot{z}_{0 x}=\ddot{\hat{x}}$, and $v_{i}(i=1,2)$ are intermediate variables used in the calculation. In absence of the input noises, and by choosing $\alpha_{0 x} \geq \lambda_{0 x}\left(L_{x}\right)^{1 / 3}>0, \alpha_{1 x} \geq \lambda_{1 x}(L)^{1 / 2}>0$ and $\alpha_{2 x} \geq \lambda_{2 x}\left(L_{x}\right)^{1 / 2}>0$, we obtain $\hat{F}_{x}=F_{x}$ after finite-time [26] with a suitable choice of the positive gains $\lambda_{0 x}, \lambda_{1 x}$ and $\lambda_{2 x}$ (see [27]).

Proof: The proof is similar to the one presented in [26]. Consider the general form of the observer:

$$
\left\{\begin{array}{l}
\dot{z}_{0}=v_{0}+U \\
v_{0}=-\lambda_{0} L^{1 /(m+1)}\left|z_{0}-\sigma\right|^{m /(m+1)} \operatorname{sign}\left(z_{0}-\sigma\right)+z_{1} \\
\dot{z}_{1}=v_{1} \\
v_{1}=-\lambda_{1} L^{1 / m}\left|z_{1}-v_{0}\right|^{(m-1) / m} \operatorname{sign}\left(z_{1}-v_{0}\right)+z_{2} \\
\ldots \\
\dot{z}_{m-1}=v_{m-1} \\
v_{m-1}=-\lambda_{m-1} L^{1 / 2}\left|z_{m-1}-v_{m-2}\right|^{(m-1) / m_{*}} \\
\quad \operatorname{sign}\left(z_{m-1}-v_{m-2}\right)+z_{m} \\
\dot{z}_{m}=-\lambda_{m} L \operatorname{sign}\left(z_{m}-v_{m-1}\right)
\end{array}\right.
$$


By introducing the notation:

$$
\left\{\begin{array}{l}
\sigma_{0}=z_{0}-\sigma \\
\sigma_{1}=z_{1}-b(t) \\
\cdots \\
\sigma_{m}=z_{m}-b^{(m-1)}(t)
\end{array}\right.
$$

and with absence of measurement noises, any solution of the above system satisfies the differential inclusion:

$$
\begin{aligned}
& \dot{\sigma}_{0}=-\lambda_{0} L^{(1 / m+1)}\left|\sigma_{0}\right|^{(m /(m+1)} \operatorname{sign}\left(\sigma_{0}\right)+\sigma_{1} \\
& \dot{\sigma}_{1}=-\lambda_{1} L^{(1 / m)}\left|\sigma_{1}-\dot{\sigma}_{0}\right|^{((m-1) / m} \operatorname{sign}\left(\sigma_{1}-\dot{\sigma}_{0}\right)+\sigma_{2} \\
& \dot{.} \\
& \dot{\sigma}_{m-1}=-\lambda_{m-1} L^{(1 / 2)}\left|\sigma_{m-1}-\dot{\sigma}_{m-2}\right|^{(1 / 2)} * \\
& \quad \operatorname{sign}\left(\sigma_{m-1}-\dot{\sigma}_{m-2}\right)+\sigma_{m} \\
& \dot{\sigma}_{m} \in-\lambda_{m} L\left(\sigma_{m}-\dot{\sigma}_{m-1}\right)+[-L, L]
\end{aligned}
$$

The derivatives of $\sigma_{i}$ are excluded here from the righthand side [28]. The obtained inclusion does not "remember" anything on the unknown signal $b(t)$ and $u(t)$ and coincides with the inclusion appearing in the proof of the Theorem 5 in [28]. Thus the proof and the choice of the parameters are the same. The rest of the proof does not differ from [28]. Thus, the convergence of our observer model for wind forces estimation (44) is validated from (47) and (51) by selecting $m=2$.

From [26], smooth second order virtual control laws can be chosen as follows:

$$
\begin{aligned}
\frac{u_{t}}{m} \hat{u}_{x} & =-z_{1 x}+K_{p}\left(V_{x}-V_{x d}\right)-K_{1 x}\left|\sigma_{x}\right|^{2 / 3} \operatorname{sign}\left(\sigma_{x}\right) \\
& -K_{2 x} \int_{0}^{t}\left|\sigma_{x}(\epsilon)\right|^{1 / 3} \operatorname{sign}\left(\sigma_{x}(\epsilon)\right) d \epsilon \\
\frac{u_{t}}{m} \hat{u}_{y} & =-z_{1 y}+K_{p}\left(V_{y}-V_{y d}\right)-K_{1 y}\left|\sigma_{y}\right|^{2 / 3} \operatorname{sign}\left(s_{y}\right) \\
& -K_{2 y} \int_{0}^{t}\left|\sigma_{y}(\epsilon)\right|^{1 / 3} \operatorname{sign}\left(\sigma_{y}(\epsilon)\right) d \epsilon
\end{aligned}
$$

where the estimation of $F_{y}$ is done by mean of $z_{1 y}$ following the same procedure. The new desired roll and pitch angles become (50), which replace those in Eq. (35).

$$
\begin{gathered}
\hat{\phi}_{r}=\arcsin \left(S_{\psi_{r}} \hat{u}_{x}-C_{\psi_{r}} \hat{u}_{y}\right) \\
\hat{\theta}_{r}=\arcsin \left(\frac{C_{\psi_{r}} \hat{u}_{x}+S_{\psi_{r}} \hat{u}_{y}}{C_{\hat{\phi}_{r}}}\right)
\end{gathered}
$$

\section{B. Controller and observer in closed loop}

It is proven that the observer under the form (44) converges in a finite time when exact measurements are available [26]. Hence, $z_{1 x}$ converges to the external disturbances $\hat{F}_{x} / m$ due to wind in the x-direction (same for $z_{1 y}$ and $\hat{F}_{y} / m$ ) in finite time. Thereafter, by substituting respectively $z_{1 x}$ and $z_{1 y}$ by $\hat{F}_{x} / m$ and $\hat{F}_{y} / m$ in (48) and (49), and by substituting the smooth controller (48) in (41), the sigma-dynamics (with $x_{1}=\sigma_{x}$ and $x_{2}=$ $\left.-\int_{0}^{t} K_{2 x}\left|x_{1}(\epsilon)\right|^{1 / 3} \operatorname{sign}\left(x_{1}(\epsilon)\right) d \epsilon\right)$ becomes:

$$
\left\{\begin{array}{l}
\dot{x}_{1}=-K_{1 x}\left|x_{1}\right|^{2 / 3} \operatorname{sign}\left(x_{1}\right)+x_{2} \\
\dot{x}_{2}=-K_{2 x}\left|x_{1}\right|^{1 / 3} \operatorname{sign}\left(x_{1}\right)
\end{array}\right.
$$

As will be proven below, this system is finite-time stable, i.e., it is asymptotically stable with finite settling time for any initial conditions and solution. Thus, the control laws developed in (48) and (49) drive $\sigma_{x}, \sigma_{y}, \dot{\sigma}_{x}$ and $\dot{\sigma}_{y}$ to zero, which defines the system motion on the sliding surface and the system actual states will converge to the desired ones.

Proof: To prove the asymptotic stability of $\sigma_{x}$ (equally $\left.\sigma_{y}\right)$, let's consider the following Lyapunov function candidate:

$$
\begin{aligned}
V & =\frac{x_{2}^{2}}{2}+\int_{0}^{x_{1}} K_{2 x}|\beta|^{1 / 3} \operatorname{sign}(\beta) d \beta \\
& =\frac{x_{2}^{2}}{2}+\frac{3}{4} K_{2 x} x_{1}^{4 / 3}
\end{aligned}
$$

By defining $\gamma=\left[\begin{array}{ll}x_{1} & x_{2}\end{array}\right]^{T}$, we obtain the first derivative of this Lyapunov function as follows:

$$
\begin{aligned}
\dot{V}= & \frac{\partial V}{\partial \gamma} \dot{\gamma} \\
= & {\left[K_{2 x}\left|x_{1}\right|^{1 / 3} \operatorname{sign}\left(x_{1}\right) \quad x_{2}\right] \cdot\left[\begin{array}{c}
x_{2}-K_{1 x}\left|x_{1}\right|^{2 / 3} \operatorname{sign}\left(x_{1}\right) \\
-K_{2 x}\left|x_{1}\right|^{1 / 3} \operatorname{sign}\left(x_{1}\right)
\end{array}\right] } \\
= & K_{2 x} x_{2}\left|x_{1}\right|^{1 / 3} \operatorname{sign}\left(x_{1}\right)-K_{1 x} K_{2 x}\left|x_{1}\right|^{1 / 3+2 / 3} \\
& -K_{2 x} x_{2}\left|x_{1}\right|^{1 / 3} \operatorname{sign}\left(x_{1}\right) \\
= & -K_{1 x} K_{2 x}\left|x_{1}\right| .
\end{aligned}
$$

By applying the LaSalle theorem, the set of $\gamma$ verifying the condition $\dot{V}(\gamma)=0$ consists of the axis $x_{1}=0$. However the unique solution that satisfies $\dot{x}_{1}=0$ in the first equation of (51) is $x_{2}=0$. So the largest invariant set is the origin $x_{1}=x_{2}=0$, thus the asymptotic stability is verified, and the variables $x_{1}$ and $x_{2}$ converge both to zero. Subsequently, it is proved in [26] that the system (51) is homogeneous (by taking $p=3$ ), in this case the asymptotic stability implies the finite-time stability of the system [29],[30],[31].

It can be noted that we use the saturation function sat instead of the sign function in both the observer and the control law in order to attenuate the chattering phenomenon which appears when the sliding variable value approaches zero, and to a obtain a smoother estimation. The expression of the function sat is given by:

$$
\operatorname{sat}(x)= \begin{cases}1 & \text { if } x>1 \\ -1 & \text { if } x<-1 \\ x & \text { otherwise }\end{cases}
$$

However, in real-world applications, false alarms may occur when the measurement of $\sigma$ terms is imprecise due to sensor noises. For this reason, we define a detection threshold for the observer, as will be shown in section V.B., and we consider the presence of wind disturbances in the system only when the absolute value of the estimated wind forces exceed this specific threshold. We chose $0.05 \mathrm{~N}$ as the threshold value for our experiments. 


\section{SimUlation AND EXPERIMENTAL VALIDATION}

The quadrotor shown in Fig. 2 is the experimental drone. We used the Raspberry pi $3+$ Navio 2 open-source autopilot and the Arducopter flight stack to perform the experimentation and to show the effectiveness of our strategy. The model parameters are given by the manufacturer in Table I:

\begin{tabular}{|c|c|c|}
\hline$K_{f}$ & Thrust factor & $2.1 * 10^{-5} \mathrm{Ns}^{2} / \mathrm{rad}^{2}$ \\
\hline$K_{t}$ & drag factor & $4 * 10^{-7} \mathrm{Nm} / \mathrm{rad}^{2}$ \\
\hline $\mathrm{m}$ & mass of the vehicle & $1.05 \mathrm{~kg}$ \\
\hline 1 & length of the arm & $0.21 \mathrm{~m}$ \\
\hline$I_{x x}, I_{y y}$ & Inertia & $3.47 * 10^{-2}{\mathrm{Kg} . \mathrm{m}^{2}}^{2}$ \\
\hline$I_{z z}$ & Inertia & $5.31 * 10^{-2} \mathrm{Kg} \cdot \mathrm{m}^{2}$ \\
\hline
\end{tabular}

TABLE I: The model's parameters

\begin{tabular}{|c|c|c|}
\hline$\lambda_{1 z}=2.5$ & $K_{1 z}=4.5$ & $K_{2 z}=4.5$ \\
\hline$\lambda_{1 x}=0.2$ & $K_{1 x}=0.5$ & $K_{2 x}=0.25$ \\
\hline$\lambda_{1 y}=0.2$ & $K_{1 y}=0.5$ & $K_{2 y}=0.25$ \\
\hline$\lambda_{1 \phi}=3.5$ & $K_{1 \phi}=4.5$ & $K_{2 \phi}=4.5$ \\
\hline$\lambda_{1 \theta}=3.5$ & $K_{1 \theta}=4.5$ & $K_{2 \theta}=4.5$ \\
\hline$\lambda_{1 \psi}=2.1$ & $K_{1 \psi}=5$ & $K_{2 \psi}=3.5$ \\
\hline
\end{tabular}

TABLE II: Control gains

The controllers parameters and the observer gains are determined empirically and given respectively in Table II and Table III.

\begin{tabular}{|c|c|c|}
\hline$\alpha_{0 x}=1$ & $\alpha_{1 x}=0.5$ & $\alpha_{2 x}=0.05$ \\
\hline$\alpha_{0 y}=1$ & $\alpha_{1 y}=0.4$ & $\alpha_{2 y}=0.045$ \\
\hline
\end{tabular}

TABLE III: Observer gains

\begin{tabular}{|c|c|}
\hline$\lambda_{1 x, y, z}=2.5,2.5,5$ & $\omega_{x, y, z}=4.1,4.1,6.8$ \\
\hline$\lambda_{2 x, y, z}=1.25,1.25,3.12$ & $\eta_{x, y, z}=1.03,1.03,3.25$ \\
\hline$\lambda_{3 x, y, z}=1.83,1.83,2.01$ & $\beta_{x, y, z}=0.5,0.5,0.8$ \\
\hline
\end{tabular}

TABLE IV: adaptive gains

In this section we will provide some simulation results to compare our proposed observer-based controller to the adaptative controller given in [32]. The adaptive controller gains wich we used in the simulations are given in the Table IV; they are associated with the adaptive controller given in [32]. We also present real experimental results to validate the effectiveness of our controller compared to the standard PID controller. Note that we didn't use the adaptative controller as a comparison in real experiments because its gains were difficult to determine even when taking the simulation gains as starting values. Comparatively, the real gains for our observer-based STA controller were easy to find and close to those used in the simulation. We thus used the PID controller as a comparison because it is still widely used and determining the gains can be done automatically using the auto-tune mode of the Arducopter.

\section{A. Simulation results (Adaptive STA vs Observer-based STA)}

In the following simulations, we compare our proposed observer-based controller to an adaptative controller in terms of robustness against wind. The simulated UAV is required to follow a circular trajectory of 3 meters radius, starting from $\xi_{i}=[0,0,0]^{T}$.

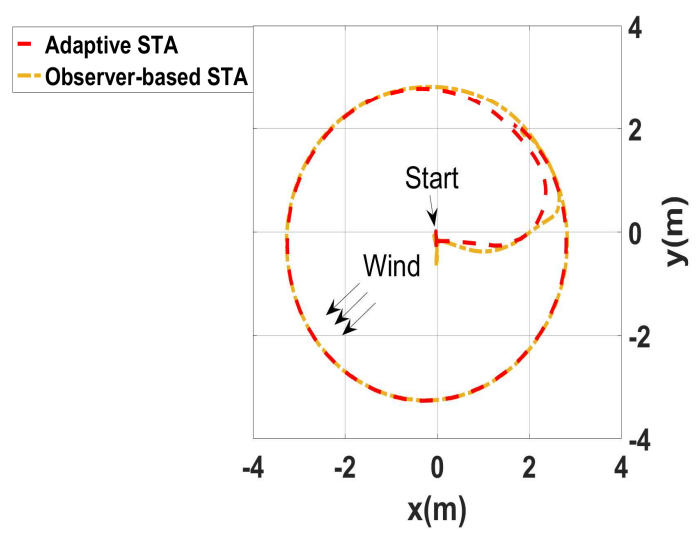

Fig. 3: Behavior of adaptive controller and observer-based controller with small wind perturbations of $0.2 \mathrm{~N}$ (Simulation)

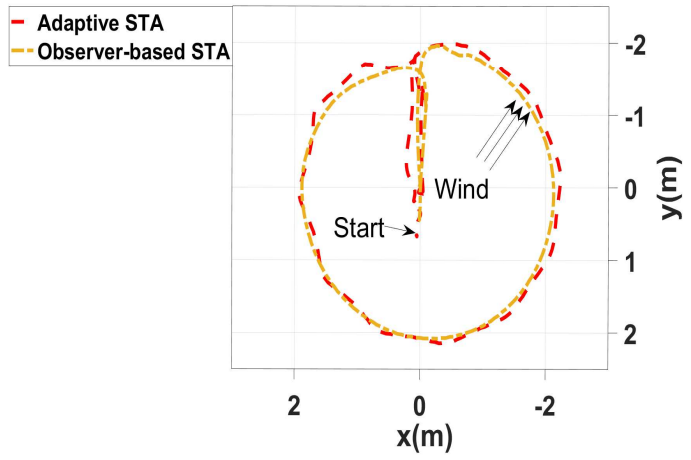

Fig. 4: Behavior of adaptive controller and observer-based controller with wind perturbations up to $5 \mathrm{~N}$ (Simulation)

In Fig. 3, we can clearly see that with small wind forces of $0.2 \mathrm{~N}$, close to what we were confronted to in real experiments, both controllers follow closely the desired trajectory. Note that in real flights, mechanical constraints, model uncertainties and sensor noises would obviously affect negatively the followed trajectory.

In the second scenario presented in Fig. 4, an external wind perturbation of $5 \mathrm{~N}$ is applied. We can notice that both controllers succeed to compensate the wind effect and maintain the requested path of the system, however the results show that the observer-based STA controller is smoother in this case. Note that such strong winds would probably be difficult to navigate for the UAV in real conditions, and that the adaptative controller had good similar results up to this wind strength. However, these simulations show more potential tolerance for our observer-based approach. By comparing the collected errors data of these simulations, given in TABLE $\mathrm{V}$, we can see that the use of the observer is an improvement over the adaptive method in terms of the robustness to the wind disturbances.

\section{B. Experimental results}

In our real experiments, an on-board GPS is used as the UAV's localization system. The quadrotor has to follow a rectilinear path starting from the initial position $\xi_{i}=$ $[0,0,1]^{T}$ as shown in the Fig. 5. The wind forces $F_{x}$ and $F_{y}$ 


\begin{tabular}{|c|c|c|}
\hline \multirow{2}{*}{} & \multicolumn{2}{|c|}{ Test 1} \\
\cline { 2 - 3 } & $\mu_{e x}(m)$ & $\mu_{e y}(m)$ \\
\hline Observer-based STA & 0.249 & 0.482 \\
\hline Adaptive STA & 0.367 & 0.597 \\
\hline & \multicolumn{2}{|c|}{ Test 2} \\
\cline { 2 - 3 } & $\mu_{e x}(m)$ & $\mu_{e y}(m)$ \\
\hline Observer-based STA & 0.5404 & 0.508 \\
\hline Adaptive STA & 0.801 & 0.673 \\
\hline
\end{tabular}

TABLE V: Position mean squared errors in x-direction $\left(\mu_{e_{x}}\right)$ and y-direction $\left(\mu_{e_{y}}\right)$ for the simulations illustrated in Fig. 3 (Test 1) and in Fig. 4 (Test 2)

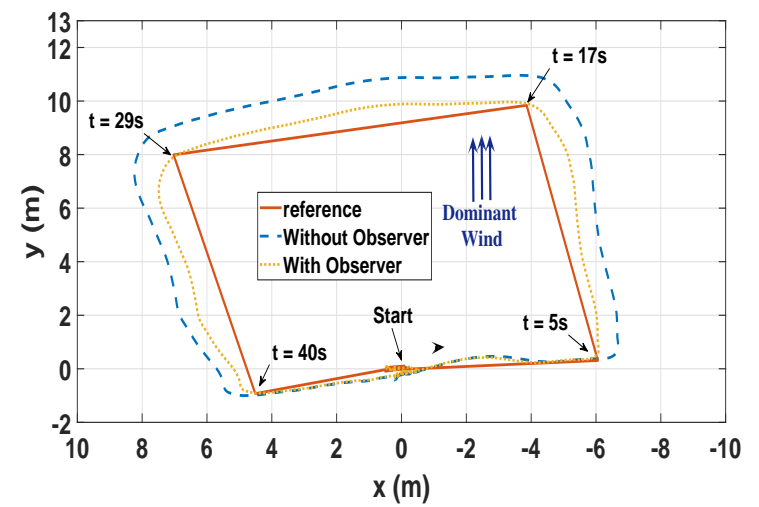

Fig. 5: Behavior of PID controller with and without Observer in the presence of wind perturbations (Experiment)

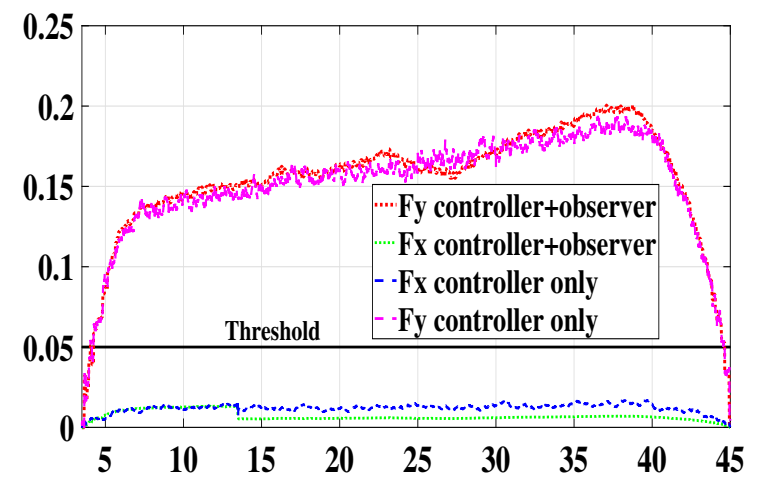

Fig. 6: Estimated wind forces $\mathrm{FX}$ and $\mathrm{FY}(\mathrm{N})$ in earth frame during the experiments (Experiment)

\begin{tabular}{|c|c|c|}
\hline \multirow{2}{*}{} & \multicolumn{2}{|c|}{ Experiment } \\
\cline { 2 - 3 } & $\mu_{e x}(m)$ & $\mu_{e y}(m)$ \\
\hline Controller with Observer & 1.519 & 1.745 \\
\hline Controller without Observer & 1.605 & 2.37 \\
\hline
\end{tabular}

TABLE VI: Position mean squared errors in $\mathrm{x}$-direction $\left(\mu_{e_{x}}\right)$ and y-direction $\left(\mu_{e_{y}}\right)$ for the experiment illustrated in Fig. 5 (in Earth-Frame) are estimated in real time by the developed observer and are presented in the Fig. 6. We can notice that the wind force is much more higher in the $\mathrm{Y}$ direction where it reaches a peak of $F_{y}=0.2 \mathrm{~N}$. This mostly explains why the PID controller drifts from the desired trajectory, as it is unable to tolerate the wind perturbations. Our observer-based controller gives much better results, although obviously not as perfect as in the simulations due to the of sensor noises and model imprecisions. Since neither the observer model nor its measurement are perfect, we used a threshold on the observer's output to avoid small perturbations due to noise values. As long as the estimated wind force stays between the threshold values, we consider it 0 , and as soon as it goes beyond them we integrate it in the control law. From indoor experiments without wind perturbations, we empirically chose threshold values of -0.05 and $0.05 \mathrm{~N}$.

In order to compare the wind conditions in both experiments, we activated the observer in both cases to estimate the wind forces, but the disturbance rejections strategy is only applied in one case (controller + observer). Fig. 6 shows that the wind forces were very similar during both experiments.

$F_{x}$ is very small and below the defined threshold, and as previously stated this estimated force is not taken into account in the controller to avoid discontinuities due to the gain tuning.

Despite the fact that the dominant wind is in the $y$ direction, the pitch and roll of the UAV are affected (see equation 43), which explains the quadrotor's positions errors on the $\mathrm{x}$ axis that we see on Fig. 5.

By comparing the means et medians of the quadrotor's positions errors in TABLE V and VI, we can see that our proposed method improved the trajectory of the quadrotor in every cases, although the adaptative method in our simulations still had pretty good results.

Note that the only theoretical drawback of the proposed strategy compared to classical controllers is an increased computational time due to the estimation of the external forces, which had no impact on our real flight experiments.

\section{CONCLUSION AND FUTURE WORKS}

The main contributions of this paper is to propose a control law and an observer based on the Super Twisting Algorithm aiming to tolerate wind perturbations and validate them on real experiments. The proposed solution guarantees the convergence of the position errors for different values and directions of external airflow attacking the vehicle. The robust controller and the nonlinear observer are both designed based on the STA to compensate for matched uncertainties such as unmodeled forces and imperfect UAV's parameters. This method was validated and compared with an adaptative controller through simulation, and a PID controller though real flights. Stability analysis of the observer controller loop has also been presented in the paper.

However, supplementary tests and improvements are needed to completely validate this method: we only tested its effectiveness against moderate wind (up to $0.2 \mathrm{~N}$ ), and we 
need to experiment in more aggressive environment where wind force can reach up to several newtons.

\section{ACKNOWLEDGMENTS}

This work was carried out in the framework of the Labex MS2T (Reference ANR-11-IDEX-0004-02) and the ROBOTEX Equipment of Excellence (Reference ANR-10- EQPX-44). It was supported by the French Government, through the program "Investments for the future" managed by the National Agency for Research and by the National Council for Scientific Research in Lebanon and the Lebanese University research support program.

\section{REFERENCES}

[1] S. Bouabdallah, "Design and control of quadrotors with application to autonomous flying," Ph.D. dissertation, 2007.

[2] S. Gupte, P. Mohandas, and J. Conrad, "A survey of quadrotor unmanned aerial vehicles," Proceedings of IEEE Southeastcon, pp. 1-6, March 2012.

[3] S. Bouabdallah, A. Noth, and R. Siegwart, "PID vs LQ control techniques applied to an indoor micro quadrotor," IEEE/RSJ International Conference on Intelligent Robots and Systems (IROS), vol. 3, pp. 2451-2456, Sept 2004.

[4] G. Hoffmann, H. Huang, S. Waslander, and C. Tomlin, "Quadrotor helicopter flight dynamics and control: Theory and experiment," Navigation and Control Conference, vol. 2, 2007.

[5] G. Raffo, M. Ortega, and F. Rubio, "An integral predictive/nonlinear $\mathrm{H}$-infinity control structure for a quadrotor helicopter," Automatica, vol. 1 , no. 46, pp. 29-39, 2010.

[6] N. Vitzilaios and N. Tsourveloudis, "An experimental test bed for small unmanned helicopters," Journal of Intelligent \& Robotic Systems, vol. 54, no. 5, pp. 769-794, 2009.

[7] A. Benallegue, A. Mokhtari, and L. Fridman, "Feedback linearization and high order sliding mode observer for a quadrotor UAV," International Workshop on Variable Structure Systems, pp. 365-372, June 2006.

[8] R. T. Palomaki, N. T. Rose, M. van den Bossche, T. J. Sherman, and S. F. De Wekker, "Wind estimation in the lower atmosphere using multirotor aircraft," Journal of Atmospheric and Oceanic Technology, vol. 34, no. 5, pp. 1183-1191, 2017.

[9] J.-Y. Wang, B. Luo, M. Zeng, and Q.-H. Meng, "A wind estimation method with an unmanned rotorcraft for environmental monitoring tasks," Sensors, vol. 18, no. 12, p. 4504, 2018.

[10] A. Martínez-Vásquez, A. Rodriguez-Mata, I. González-Hernández, S. Salazar, A. Montiel-Varela, and R. Lozano, "Linear observer for estimating wind gust in uav's," in Electrical Engineering, 12th International Conference on Computing Science and Automatic Control (CCE), 2015, pp. 1-6.

[11] K. Alexis, G. Nikolakopoulos, and A. Tzes, "Experimental model predictive attitude tracking control of a quadrotor helicopter subject to wind-gusts," 18th Mediterranean Conference on Control \& Automation (MED), pp. 1461-1466, 2010.

[12] N. Sydney, B. Smyth, and D. A. Paley, "Dynamic control of autonomous quadrotor flight in an estimated wind field," IEEE 52nd Annual Conference on Decision and Control (CDC), pp. 3609-3616, 2013.

[13] N. K. Tran, E. Bulka, and M. Nahon, "Quadrotor control in a wind field," International Conference on Unmanned Aircraft Systems (ICUAS), pp. 320-328, 2015.

[14] H. Huang, G. M. Hoffmann, S. L. Waslander, and C. J. Tomlin, "Aerodynamics and control of autonomous quadrotor helicopters in aggressive maneuvering," IEEE International Conference on Robotics and Automation (ICRA), pp. 3277-3282, 2009.

[15] F. Léonard, A. Martini, and G. Abba, "Robust nonlinear controls of model-scale helicopters under lateral and vertical wind gusts," IEEE Transactions on Control Systems Technology, vol. 20, no. 1, pp. 154$163,2012$.

[16] J. I. Vasquez-Gomez, C. Gomez-Castañeda, E. M. De Cote, and J. C. Herrera-Lozada, "Multirotor uav coverage planning under wind conditions," International Conference on Mechatronics, Electronics and Automotive Engineering (ICMEAE), pp. 32-37, 2016.

[17] W. Dong, G.-Y. Gu, X. Zhu, and H. Ding, "High-performance trajectory tracking control of a quadrotor with disturbance observer," Sensors and Actuators A: Physical, vol. 211, pp. 67-77, 2014.
[18] S. Karpenko, I. Konovalenko, A. Miller, B. Miller, and D. Nikolaev, "Stochastic control of uav on the basis of robust filtering of $3 \mathrm{~d}$ natural landmarks observations," Proceedings of the 39th IITP RAS Interdisciplinary Conference \& School, pp. 7-18, 2015.

[19] C. Nicol, C. Macnab, and A. Ramirez-Serrano, "Robust adaptive control of a quadrotor helicopter," Mechatronics, vol. 21, no. 6, pp. 927-938, 2011.

[20] J. Escareño, S. Salazar, H. Romero, and R. Lozano, "Trajectory control of a quadrotor subject to $2 \mathrm{~d}$ wind disturbances," Journal of Intelligent \& Robotic Systems, vol. 70, no. 1-4, pp. 51-63, 2013.

[21] C. Wang, B. Song, P. Huang, and C. Tang, "Trajectory tracking control for quadrotor robot subject to payload variation and wind gust disturbance," Journal of Intelligent \& Robotic Systems, vol. 83, no. 2, pp. 315-333, August 2016.

[22] L. Derafa, A. Benallegue, and L. Fridman, "Super twisting control algorithm for the attitude tracking of a four rotors UAV," Journal of the Franklin Institute, vol. 349, no. 2, pp. 685-699, 2012.

[23] L. Luque-Vega, B. Castillo-Toledo, and A. Loukianov, "On the quadrotor trajectory tracking problem via super twisting technique," World Automation Congress (WAC), pp. 1-6, 2012.

[24] J. Kasac, S. Stevanovic, T. Zillic, and J. Stepanic, "Robust output tracking control of a quadrotor in the presence of external disturbances," Transactions of FAMENA, pp. 29-42, 2014.

[25] S. Bouabdallah, P. Murrieri, and R. Siegwart, "Design and control of an indoor micro quadrotor," IEEE International Conference on Robotics and Automation (ICRA), vol. 5, pp. 4393-4398, 2004.

[26] Y. B. Shtessel, I. A. Shkolnikov, and A. Levant, "Smooth second-order sliding modes: Missile guidance application," Automatica, vol. 43, no. 8, pp. 1470 - 1476, 2007.

[27] J. Davila, L. Fridman, and A. Levant, "Second-order sliding-mode observer for mechanical systems," IEEE Transactions on Automatic Control, vol. 50, no. 11, pp. 1785-1789, Novembre 2005.

[28] A. Levant, "Higher-order sliding modes, differentiation and outputfeedback control," International journal of Control, vol. 76, no. 9-10, pp. 924-941, 2003.

[29] A. Bacciotti and L. Rosier, Liapunov functions and stability in control theory. Springer, Lecture Notes in Control and Information Sciences, 2001.

[30] S. P. Bhat and D. S. Bernstein, "Finite-time stability of continuous autonomous systems," SIAM Journal on Control and Optimization, vol. 38, no. 3, pp. 751-766, 2000.

[31] A. Levant, "Homogeneity approach to high-order sliding mode design," Automatica, vol. 41, no. 5, pp. 823-830, 2005.

[32] S. Rajappa, C. Masone, H. H. Bülthoff, and P. Stegagno, "Adaptive super twisting controller for a quadrotor uav," IEEE International Conference on Robotics and Automation (ICRA), pp. 2971-2977, 2016. 